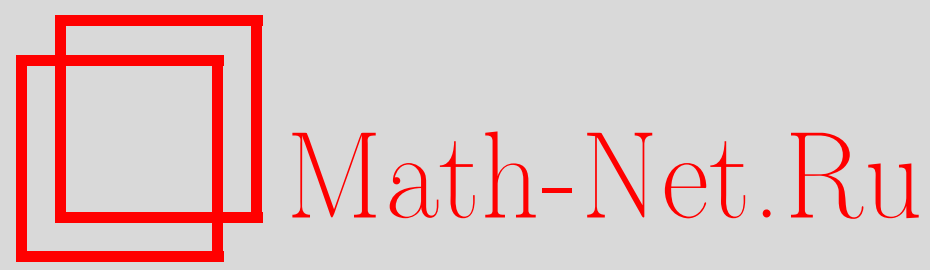

И. В. Соболев, О бирациональной жесткости одной серии многомерных Фано-расслоений, Матем. заметки, 2000, том 68, выпуск 3, 455-460

DOI: https://doi.org/10.4213/mzm963

Использование Общероссийского математического портала Math-Net.Ru подразумевает, что вы прочитали и согласны с пользовательским соглашением http://www.mathnet.ru/rus/agreement

Параметры загрузки:

IP : 54.89 .56 .158

26 апреля 2023 г., 15:03:44

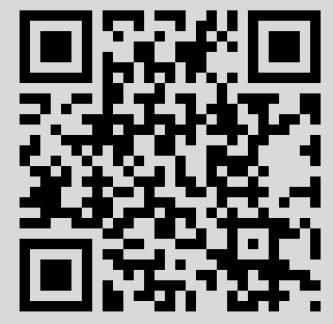




\title{
О БИРАЦИОНАЛЬНОЙ ЖЕСТКОСТИ ОДНОЙ СЕРИИ МНОГОМЕРНЫХ ФАНО-РАССЛОЕНИЙ
}

\section{И. В. Соболев}

\begin{abstract}
В работе доказана бирациональная жесткость алгебраических многообразий с пучком гиперповерхностей степени $M$ в $\mathbb{P}^{M}$, которые имеют степень 2 по базе.

Библиографиял: 9 названий.
\end{abstract}

1. Введение. Одним из наиболее мощных методов современной бирациональной геометрии является метод максимальных особенностей, созданньй В.А. Исковских и Ю.И. Маниньпм (см. [1]). В своей первоначальной форме он, однако, имел довольно узкую область применения. Недавние технические усовершенствования, введенные А.В. Пухликовым, позволили сушественно расширить ее, в частности, стало возможным описание бирациональных автоморфизмов многообразий произвольной размерности.

В работах [2]-[4] установлена бирациональная жесткость некоторых классов Фанорасслоений, удовлетворяющих так называемому $K^{2}$-условию. Для определения гранищ применимости метода максимальных особенностей полезно рассматривать примеры многообразий, для которых это условие не выполнено. Один из них разбирается в [5]. В настоящей работе исследуется другой пример подобного рода.

Пусть $V_{m}$ - гиперповерхность в $\mathbb{P}^{1} \times \mathbb{P}^{M}$, определенная уравнением

$$
p_{m, M}\left(u, v ; x_{0}, \ldots, x_{M}\right)=0,
$$

где $p$ имеет степень $m$ по $u, v$ и степень $M$ по $x_{0}, \ldots, x_{M}$. Очевидным образом определена проекция $\pi: V \rightarrow \mathbb{P}^{1}$, слоем которой является гиперповерхность степени $M$ в $\mathbb{P}^{M}$. Всюду в дальнейшем $F$ будет обозначать слой $\pi$.

В [2], [3] доказано, что при $m \geqslant 3$ и $M \geqslant 4$ общее $V_{m}$ бирационально сверхжестко и обладает единственной структурой $Ф$ ано-расслоения. С другой стороны, $V_{1}$ рационально при всех $M$. Вопрос о рациональности многообразия $V=V_{2}$ в этих работах оставлен открытым.

Многообразие $V$ имеет вторую структуру Фано-расслоения. Действительно, пусть $\sigma: V \rightarrow \mathbb{P}^{M}$ - проекция на второй множитель. Тогда отображение $\tau: V \rightarrow V$, которое переставляет точки в слоях $\sigma$, определено вне подмножества коразмерности 2 и является бирациональной инволюцией. Вторая структура получается действием $\tau$ на первую.

Работа выполнена при финансовой поддержке Российского фонда фундаментальных исследований, грант № 99-01-01132, и фонда поддержки научных школ, грант № 96-15-96146. 
ГипотеЗА 1. При $M \geqslant 4$ и общем $V$ группа $\operatorname{Bir} V$ состоит из двух элементов и порождается $\tau$; на $V$ имеется только две структуры расслоения на рационально связные многообразия.

ГиПотеЗА 2. При $M=3 \operatorname{Bir} V$ свободно порождается $\tau$ и групшой $\operatorname{Bir} F_{\eta}$, где $F_{\eta}-$ слой $\pi$ над общей точкой $\mathbb{P}^{1}$. Любой пучок рациональных поверхностей на $V$ бирациональными преобразованиями можно перевести в $|F|$.

Гипотеза 2 косвенно подтверждается результатами Барделли [6].

2. Формулировка результата. Прежде чем сформулировать наш основной результат, напомним основные понятия метода максимальных особенностей.

Порогом канонического присоединения линейной системы $D$ на $Ф$ ано-расслоении $\rho$ : $V \rightarrow S$ называется число

$$
c(D, W)=\sup \left\{\frac{b}{a}\left|a, b \in \mathbb{Z}_{+},\right| a D+b K_{V} \mid \neq \varnothing\right\} .
$$

Фано-расслоение $\rho: V \rightarrow S$ назьвается бирационально жестким, если для любого Фано-расслоения $\rho^{\prime}: V^{\prime} \rightarrow S^{\prime}, \operatorname{dim} V^{\prime}=\operatorname{dim} V$, бирационального отображения $\chi:$ $V \rightarrow V^{\prime}$ и линейной системы $\left|D^{\prime}\right|$ на $V^{\prime}$, свободной в коразмерности 1 , существует бирациональньй автоморфизм $\chi^{\prime}$ многообразия $V$ такой, что $c(D, V) \leqslant c\left(D^{\prime}, V^{\prime}\right)$, где $|D|=\left(\chi \circ \chi^{\prime}\right)^{-1}\left|D^{\prime}\right|$.

Докажем следующий результат.

ТЕОремА. Если многообразие $V$ удовлетворяет условиям сильной регулярности, приведенным ниже, то оно бирационально жестко при $M \geqslant 6$. Группа $\operatorname{Bir} V$ для общего $V$ изоморфна $\mathbb{Z}_{2}$ и порождается $\tau$.

СлЕдСТВия.

1) $V$ обладает лишь двумя структурами Фано-расслоения.

2) Многообразие $V$ не рачионально.

Перейдем к нужным нам условиям общности.

Рассмотрим гиперповерхность $W$ степени $M$ в $\mathbb{P}^{M}$. Пусть $p$ - произвольная точка $W$ и в аффинной системе координат с центром в $p$ уравнение $W$ записьвается в виде $q_{1}+q_{2}+\cdots+q_{M}=0, q_{i}$ - однородньй многочлен степени $i$.

ОПРЕДЕЛЕНИЕ. Гладкую точку $p \in W$ назовем сильно регулярной, если последовательность $\left(q_{1}, \ldots, q_{M-1}\right)$ регулярна и, кроме того, уравнения $q_{2}=\cdots=q_{M}=0$ определяют в проективном пространстве $\mathbb{P}^{M-1}$ конечное множество точек, причем сумма кратностей всех точек, лежащих в гиперплоскости $q_{1}=0$, не превосходит $M$.

Будем говорить, что Фано-расслоение $V$ сильно регулярно, если каждая гладкая точка любого слоя проекции $\pi$ сильно регулярна, а каждая особая - регулярна в смысле работы [3]. С помощью счета констант можно показать, что общее $V$ является сильно регулярньм (см. [3], [7]).

3. Максимальные особенности. Пусть теперь $\chi: V \rightarrow\left(W, D^{\prime}\right)$ - бирациональное отображение, $|\chi|=D=\chi^{-1}\left(D^{\prime}\right), D=-n K_{V}+l F$. Предположим, что $l<0$. Легко видеть, что $\tau$ действует на $\operatorname{Pic} V$ следующим образом:

$$
\tau^{*} K_{V}=K_{V}, \quad \tau^{*} F=\sigma^{-1}(\sigma(F))-F=M K_{V}-F,
$$


т. е. $(\chi \circ \tau)^{-1}\left(D^{\prime}\right)=-n K_{V}+l\left(M K_{V}-F\right)=-n^{\prime} K_{V}+l^{\prime} F$, где $l^{\prime}>0$.

Допустим, что $c(D, V)>c\left(D^{\prime}, W\right)$. В силу сказанного вьше можно считать, что $l>0$.

Дискретное нормирование $\nu$ поля рациональных функций многообразия $V$ назьвается максимальной особенностью, если выполняется неравенство Нётера-Фано-Исковских $e(\nu)=\nu(|\chi|)-n K(\nu, V)>0$, где $K(\nu, V)-$ дискрепантность $\nu$ по отношению к $V$.

Так же, как в [2], [3], доказывается, что если $c(D, V)>c\left(D^{\prime}, W\right)$, то центры всех максимальных особенностей лежат в слоях $\pi$ и существует конечное множество максимальных особенностей $\mathscr{M}$ таких, что вьполнено следующее неравенство:

$$
\sum_{t \in \mathbb{P}^{1}} \max _{\left\{\nu \in \mathscr{M} \mid \operatorname{centre}(\nu) \subset F_{t}\right\}}\left(\frac{e(\nu)}{\nu\left(F_{t}\right)}\right)>l
$$

где $F_{t}=\pi^{-1}(t)$.

ЛЕмма 1. Пусть $g$ - гиперплоское сечение $F$. Тогда если иикл $N K_{V}^{2}-r g$ әффективен, то $r<2 N / M$.

ДокАЗАТЕЛЬСтво. Пусть $L_{V}$-ограничение тавтологического пучка $L \in \operatorname{Pic} \mathbb{P}^{1} \times \mathbb{P}^{M}$ на $V$. Так как линейная система $\left|L_{V}\right|$ свободна, то $\left(N K_{V}^{2}-r g\right) L_{V}^{M-2}>0$, если $N K_{V}^{2}-r g$ эффективен. Имеем

$$
K_{V}=-L_{V}, \quad\left(N K_{V}^{2}-r g\right) L_{V}^{M-2}=N L_{V}^{M}-r L_{V}^{M-1} F=2 N-r M>0,
$$

т.e. $r<2 N / M$.

Пусть $D_{i} \in|D|$ - общие дивизоры и $Z=\left(D_{1} \circ D_{2}\right), Z=Z^{v}+Z^{h}$, где $Z^{h}$ накрьвает базу, а компоненты $Z^{v}$ лежат в слоях;

$$
\begin{gathered}
Z=Z^{h}+\sum_{t \in \mathbb{P}^{1}} Z_{t}^{v}, \quad \operatorname{supp} Z_{t}^{v} \subset F_{t}, \\
Z=\left(D_{1} \circ D_{2}\right) \equiv n^{2} K_{V}^{2}-2 n l K_{V} F=n^{2} K_{V}^{2}+2 n l g .
\end{gathered}
$$

Так как $Z-\sum_{t \in \mathbb{P}^{1}} Z_{t}^{v}$ эффективен, то $\sum_{t \in \mathbb{P}^{1}} \operatorname{deg} Z_{t}^{v}-2 n l M<2 n^{2}$ или

$$
\sum_{t \in \mathbb{P}^{1}} \operatorname{deg} Z_{t}^{v}<2 n^{2}+2 M n \sum_{t \in \mathbb{P}^{1}} \max _{\left\{\nu \in \mathscr{M} \mid \operatorname{centre}(\nu) \subset F_{t}\right\}}\left(\frac{e(\nu)}{\nu\left(F_{t}\right)}\right)
$$

Отсюда существует максимальная особенность $\nu \in \mathscr{M}$ такая, что centre $(\nu) \subset F=F_{t}$ и

$$
\nu(F) \operatorname{deg} Z_{t}^{v}<2 n^{2} \nu(F)+2 M n e(\nu) .
$$

Приходим к противоречию существования такой максимальной особенности. 
4. Оценки кратностей. Пусть $\varphi_{i, i-1}: V_{i} \rightarrow V_{i-1}, i=1, \ldots, K,-$ разрешение дискретного нормирования $\nu$ (подробности см. в [7], [8]), где $\varphi_{i, i-1}-$ раздутие с центром $B_{i-1} \subset V_{i-1}$ и исключительным дивизором $E_{i} \subset V_{i}$, причем первые $L \leqslant K$ раздутий соответствуют циклам $B_{i-1}$ коразмерности $>3$, а первые $N \leqslant L$ - циклам $B_{i-1} \subset F^{i-1}$ (верхний индекс обозначает собственньй прообраз относительно соответствующего раздутия). На множестве индексов $\{1, \ldots, K\}$ введем структуру ориентированного граффа: $j$ и $i$ соединяются стрелкой, если $j>i$ и $B_{j-1} \subset E_{i}^{j-1}$. Пусть $p_{i}-$ число путей в этом графе, ведущих из вершины $K$ в вершину $i$. Обозначим

$$
\Sigma_{l}=\sum_{i=1}^{L} p_{i}, \quad \Sigma_{u}=\sum_{i=L+1}^{K} p_{i}, \quad \Sigma_{f}=\sum_{i=1}^{N} p_{i}, \quad d_{h}=\operatorname{deg} Z^{h}, \quad d_{v}=\operatorname{deg} Z^{v},
$$

$m_{i}^{h}=\operatorname{mult}_{B_{i}-1}\left(Z^{h}\right)^{i-1}, \quad m_{i}^{v}=\operatorname{mult}_{B_{i}-1}\left(Z^{v}\right)^{i-1}, \quad m_{i}^{h} \leqslant m_{1}^{h}=m^{h}, \quad m_{i}^{v} \leqslant m_{1}^{v}=m^{v}$.

В следующих двух леммах мы используем элементарную теорию пересечений (см., например, [9]).

Лемма 2. Пусть $p \in F-$ сильно регулярная гладкая точка $u Z \subset F-$ эффективный иикл коразмерности 2. Тогда при $M \geqslant 6$ верно неравенство

$$
\frac{\operatorname{mult}_{p} Z}{\operatorname{deg} Z} \leqslant \frac{3}{M} \text {. }
$$

ДокАЗАТЕльство. Пусть $T_{p} F-$ касательная гиперплоскость к $F$ в $p$.

Рассмотрим два случая.

1) $Z$ не содержится в $T_{p} F \cap F=T$. Тогда $(Z \circ T)$ - эффективньй цикл коразмерности 3 на $F$ и по доказанному в [7]

$$
\frac{5}{2 M} \geqslant \frac{1}{2} \frac{\operatorname{mult}_{p}(Z \circ T)}{\operatorname{deg}(Z \circ T)} \geqslant \frac{\operatorname{mult}_{p} Z}{\operatorname{deg} Z} .
$$

2) $Z \subset T$. В этом случае мы рассуждаем, как в лемме 6 из [3].

Пусть $Q$ - квадратичный касательный конус к $T$ в точке $p$ и $S=Q \cap T$. Для того чтобы получить нужное неравенство, нужно показать, что $S$ заметается семейством кривьх $C_{t}$, общий член которого неприводим и удовлетворяет неравенству

$$
\frac{\operatorname{mult}_{p} C_{t}}{\operatorname{deg} C_{t}}>\frac{2}{3} \text {. }
$$

Рассмотрим линейные системы $\Lambda_{k}=|k H-(k+1) E|, k=2, \ldots, M-1$, где $H$ - гиперплоское сечение $T$, а $E$ - исключительный дивизор раздутия точки $p$. Так как $p-$ сильно регулярная особая точка, то $\operatorname{codim}_{T} B s \Lambda_{k} \geqslant k-1$; следовательно, для общих дивизоров $D_{k} \in \Lambda_{k}$ семейство одномерных циклов

$$
C^{*}=\left(S \circ D_{4} \circ \cdots \circ D_{M-1}\right)
$$

состоит из эффективных кривых. Общий член семейства $C^{*}$ не обязательно неприводим, потому что $C^{*}$ может содержать прямые, проходяшие через $p$, среди которых могут быть и кратные. Выбросив эти прямые, получим семейство $C$, все кривые которого удовлетворяют неравенству

$$
\frac{\operatorname{mult}_{p} C}{\operatorname{deg} C} \geqslant \frac{M ! / 4-\gamma_{1}}{M ! / 3-\gamma_{2}}
$$


где

$$
0 \leqslant \gamma_{2} \leqslant \gamma_{1} \leqslant M
$$

так как выполнено условие сильной регулярности, т.е. неравенство

$$
\frac{\operatorname{mult}_{p} C}{\operatorname{deg} C}>\frac{2}{3}
$$

выполняется при $M \geqslant 6$.

Лемма 3. Если $p$ - регулярная особая точка $F, Z$ - әффективный иикл коразмерности 2 на $V$, не содержашийся в $F$, то

$$
\frac{\operatorname{mult}_{p} Z}{\operatorname{deg} Z} \leqslant \frac{2}{M}
$$

ДокАЗАТЕЛьСТво. По доказанному в [3] имеем

$$
\frac{4}{M} \geqslant \frac{\operatorname{mult}_{p}(Z \circ F)}{\operatorname{deg}(Z \circ F)} \geqslant 2 \frac{\operatorname{mult}_{p} Z}{\operatorname{deg} Z} .
$$

Лемма 4. Для любого простого дивизора $Y$ и неособой точки $p \in F$ верно неравенство

$$
\frac{\operatorname{mult}_{p} Y}{\operatorname{deg} Y} \leqslant \frac{2}{M} \text {. }
$$

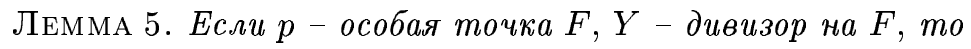

$$
\frac{\operatorname{mult}_{p} Y}{\operatorname{deg} Y} \leqslant \frac{3}{M} \text {. }
$$

Лемма 6. Пусть $p$ - особая точка $F, Y$ - дивизор на $F, \tilde{Y}$ - его собственный прообраз при раздутии точки $p, x$ - точка исключительного дивизора раздутия. Тогда выполняется неравенство

$$
\frac{\operatorname{mult}_{x} \tilde{Y}}{\operatorname{deg} Y} \leqslant \frac{2}{M}
$$

Доказательство лемм 4-6 см. в [3].

5. Исключение максимальной особенности. Так же, как в [2], мы получаем квадратичное неравенство

$$
\left(\Sigma_{l}+\Sigma_{u}\right)\left(\sum_{i=1}^{L} p_{i} m_{i}^{h}+\sum_{i=1}^{N} p_{i} m_{i}^{v}\right) \geqslant\left(2 \Sigma_{l}+\Sigma_{u}\right)^{2} n^{2}+2\left(2 \Sigma_{l}+\Sigma_{u}\right) n e+e^{2} .
$$

Рассмотрим два случая. 
1) Общая точка цикла $B_{0}=\operatorname{centre}(\nu)$ неособа на $F$. Тогда при $M \geqslant 6$

$$
\begin{gathered}
m^{h} \leqslant \frac{3}{M} d_{h}=3 n^{2}, \quad m^{v} \leqslant \frac{2}{M} d_{v} \\
d_{v} \leqslant 2 n^{2}+2 M n \frac{e(\nu)}{\nu(F)} \leqslant 2 n^{2}+\frac{2 M n e}{\Sigma_{f}} \\
\sum_{i=1}^{L} p_{i} m_{i}^{h}+\sum_{i=1}^{N} p_{i} m_{i}^{v} \leqslant m^{h} \Sigma_{l}+m^{v} \Sigma_{f} \leqslant 3 n^{2} \Sigma_{l}+\frac{4 n^{2}}{M} \Sigma_{f}+4 n e .
\end{gathered}
$$

Из квадратичного неравенства имеем

$$
n^{2}\left(\Sigma_{l}+\Sigma_{u}\right)\left(\frac{4}{M} \Sigma_{f}-\Sigma_{l}\right) \geqslant\left(n \Sigma_{u}-e\right)^{2} .
$$

Так как $\Sigma_{f} \leqslant \Sigma_{l}$, то получаем противоречие.

2) $B_{0}=\operatorname{centre}(\nu)=p$ - особая точка $F$. Положим $\Sigma_{f}^{\prime}=2 p_{1}+\sum_{i=2}^{N} p_{i}, \Sigma_{f}^{\prime} \leqslant 2 \Sigma_{l}$. Имеем

$$
\begin{gathered}
m^{h} \leqslant \frac{2}{M} d_{h}=2 n^{2}, \quad m_{1}^{v} \leqslant \frac{3}{M} d_{v}<\frac{4}{M} d_{v}, \quad m_{i}^{v} \leqslant \frac{2}{M} d_{v} \quad \text { при } i \geqslant 2 \\
d_{v} \leqslant 2 n^{2}+2 M n \frac{e(\nu)}{\nu(F)} \leqslant 2 n^{2}+\frac{2 M n e}{\Sigma_{f}^{\prime}} \\
\sum_{i=1}^{L} p_{i} m_{i}^{h}+\sum_{i=1}^{N} p_{i} m_{i}^{v} \leqslant m^{h} \Sigma_{l}+p_{1} m_{1}^{v}+\sum_{i=2}^{N} p_{i} m_{i}^{v} \leqslant 2 n^{2} \Sigma_{l}+\frac{4 n^{2}}{M} \Sigma_{f}^{\prime}+4 n e \\
n^{2}\left(\Sigma_{l}+\Sigma_{u}\right)\left(\frac{4}{M} \Sigma_{f}^{\prime}-2 \Sigma_{l}\right) \geqslant\left(n \Sigma_{u}-e\right)^{2} .
\end{gathered}
$$

Противоречие при $M>4$. Доказательство основной теоремы закончено.

Автор благодарит А.В. Пухликова за постановку и обсуждение проблемы.

\section{СПИСОК ЦИТИРОВАННОЙ ЛИТЕРАТУРЫ}

[1] Пухликов А. В. Бирациональные автоморфизмы трехмерных алгебраических многообразий с пучком поверхностей Дель Пеццо // Изв. РАН. Сер. матем. 1998. Т. 62. № 1. С. 123-164.

[2] Пухликов А. В. Бирационально жесткие расслоения Фано // Изв. РАН. Сер. матем. 2000. T. 64. № 3. C. 131-150.

[3] Pukhlikov A. V. Certain examples of birationally rigid varieties with a pencil of double quadrics. MPI preprint 1998-15.

[4] Гриненко М. М. Бирациональные автоморфизмы трехмерного двойного конуса // Матем. сб. 1998. Т. 189. № 7. С. 37-52.

[5] Pukhlikov A. V. Birational automorphisms of Fano hypersurfaces // Invent. Math. 1998. V. 134. P. 401-426.

[6] Фултон У. Теория пересечений. М.: Мир, 1989.

[7] Pukhlikov A. V. Essentials of method of maximal singularities. Warwick preprint 31-1996.

[8] Bardelli F. Polarized mixed Hodge structures: On irrationality of threefolds via degeneration // Ann. Mat. Pura et Appl. 1984. V. 137. P. 287-369.

[9] Исковских В. А., Манин Ю. И. Трехмерные квартики и контрпримеры к проблеме Люрота // Матем. сб. 1971. Т. 86(128). №1. С. 140-166. 